\title{
Primary Adrenal Insufficiency
}

National Cancer Institute

\section{Source}

National Cancer Institute. Primary Adrenal Insufficiency. NCI Thesaurus. Code 6113172.

A hormonal disorder that occurs when the adrenal glands fail to release adequate amounts of glucocorticoids (cortisol), mineralocorticoids (aldosterone, 11-

deoxycorticosterone), and androgens (dehydroepiandrosterone) to meet physiologic needs, despite release of ACTH from the pituitary. 